\title{
Two software tools for facilitating the choice of ground source heat pumps by stakeholders and designers
}

\author{
Michele De Carli*1, Amaia Castelruiz Aguirre ${ }^{2}$, Angelo Zarrella ${ }^{1}$, Lucia Cardoso $^{3}$, Sarah Noyé $^{2}$, Robert Gast ${ }^{3}$, Samantha \\ Graci $^{l}$, Giuseppe Emmi ${ }^{l}$, David Bertermann ${ }^{4}$, Johannes Müller ${ }^{4}$, Antonio Galgaro ${ }^{5}$, Giorgia Dalla Santa ${ }^{5}$ Fabio Poletto ${ }^{6}$, \\ Giulia Mezzasalma ${ }^{7}$, Silvia Contini ${ }^{7}$, Javier Urchueguía ${ }^{8}$, Marco Belliardi $^{9}$, Riccardo asquali $^{10}$, Adriana Bernardi $^{11}$
}

\footnotetext{
${ }^{1}$ Department of Industrial Engineering, University of Padua, Italy

${ }^{2}$ Fundacion Tecnalia Research and Innovation, Spain

${ }^{3}$ Aner Sistemas Informaticos, S.L.

${ }^{4}$ Friedrich-Alexander Universitaet Erlangen-Nuernberg, Germany

${ }^{5}$ Depatment of Geosciences, University of Padua, Italy

${ }^{6}$ Galletti Belgium NV, Belgium

${ }^{7}$ Red Srl, Italy

${ }^{8}$ Universidad Politecnica de Valencia, Spain

${ }^{9}$ SUPSI, Switzerland

${ }^{10}$ Slr Environmental Consulting Ltd, Ireland

${ }^{11}$ CNR-ISAC, Italy
}

\begin{abstract}
For promoting the diffusion of GSHP and making the technology more accessible to the general public, in the H2020 research project "CHeap and Efficient APplication of reliable Ground Source Heat exchangers and PumpS" (acronym Cheap-GSHPs) a tool for sizing these systems has been developed, as well as a Decision Support System (DSS) able to assist the user in the preliminary design of the most suitable configuration.

For all these tools a common platform has been carried out considering climatic conditions, energy demand of buildings, ground thermal properties, heat pump solutions repository, as well as renewable energy database to use in synergy with the GSHPs. Since the aims of the tools are different, there are different approaches.

The design tool is mainly addressed to designers. The calculation may be done in two ways: with a simplified method based on the ASHRAE approach and with a detailed calculation based on the numerical tool CaRM (Capacity-Resistance method).

The DSS final aim is to support decision-making, by providing the stakeholders at all the level with a series of scenario. The Cheap-GSHPs project has developed a DSS tool aimed at accelerating the decision-making process of designers and building owners as well as increasing market share of the Cheap-GSHPs technologies. Hence the DSS generates different possible solutions based on a defined general problem, identifying the optimal solution.

Both tools are presented in the paper, showing the potentialities provided by both software.
\end{abstract}

\section{Introduction}

A big obstacle for the diffusion of GSHPs is on one hand the lack of knowledge of people and stakeholders on the technology and on the other hand the inexperience of designers in sizing the Ground Heat Exchangers (GHEs) field due to a lack of knowledge. This means that people can be divided into different classes: technical and non-technical stakeholders. Technical stakeholders are designers, architects and researchers. Non-technical stakeholders are public administrations, general investors, end users. Usually these two types of stakeholders have different backgrounds and different purposes, hence different approaches have to be followed in order to meet their needs and try to get them reaching their goals.

For promoting the diffusion of GSHP and making the technology more accessible to the general public, in the research project "CHeap and Efficient APplication of reliable Ground Source Heat exchangers and PumpS" (acronym Cheap-GSHPs) hence two software have been developed in order to allow both types of stakeholders to get more confident with the GSHP technology. The first one is a Decision Support System (DSS) able to assist the user in the preliminary design of the most suitable configuration (named GeoHP-DSS) and the second one is tool for sizing GHEs (named GeoHPDesign).

* Corresponding author: michele.decarli@unipd.it 
Turban et al. [1] broadly define a DSS as: "a computerbased information system that combines models and data in attempt to solve semi-structured and some unstructured problems with extensive user involvement". This information system requires hardware and software components plus a series of human elements such as designers and end-users to live. The system's final aim is to support decision-making, by providing the stakeholders at all the level with a series of scenario. The Cheap-GSHPs project has developed a DSS tool aimed at accelerating the decision-making process of designers and building owners as well as increasing market share of the CheapGSHPs technologies. Hence the DSS generates different possible solutions based on a defined general problem, identifying the optimal solution. This means that the user defines few and simple inputs (type of building, overall floor area or gross volume, location) to generate a first cost-benefit analysis and to check the feasibility of the GSHP solution. The DSS needs a consistent set of databases and simplified calculation methods for generating the different possible solutions. For evaluating the overall length of the GHE field the DSS needs a set of energy demands of the buildings in the different climatic locations.

On the other hand, the design tool is mainly addressed to designers. The calculation may be done in two ways: with a simplified method based on the ASHRAE approach [2] and with a detailed calculation based on the numerical tool CaRM [3].

In the paper first the common platform and databases will be presented. Then the DSS and the design tool will be described and discussed.

\section{The common platform}

In order to meet the needs of both the DSS and the design tool, suitable databases have to be collected and a common platform has to be carried out (Figure 1).

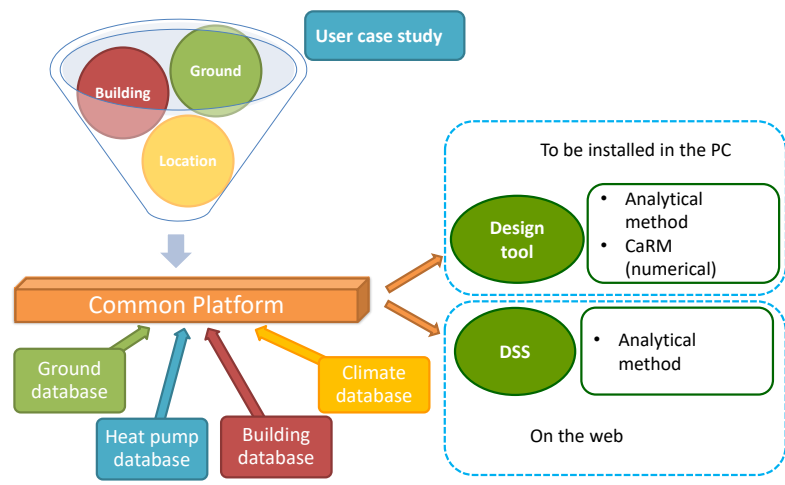

Fig. 1. Main philosophy of the DSS Tool and the Design Tool

The input definition is different in the two tools. In GeoHP-DSS the input of the user are few and, for getting proper calculations, the databases and the calculations have to be simple. For GeoHP-Design the input are mostly provided by the user, but a set of possible default values and input may be provided by the tool in order to help the user and facilitate in the sizing procedure. Hence common values are used for the two tools, even though there might be slightly differences in the databases or in the calculation procedures. In any case the methodology is common. The databases which have been set up during the project are:

- The ground databases are based on PARMADO and PARMADO1 [4] as well as on additional work for defining the thermal properties of ground materials based on literature review and on measurements [5]

- For the climatic database a database based on different TRY has been built up as well as classification on the Köppen-Geiger scale [6]

- The building database has been set for having predefined heating and cooling yearly energy demands of buildings as well as hourly average profiles of loads for each month [7]

- Heat pumps are based on three different sets of data. The first one (used in the DSS) is based on a specific tool developed in the project. The second one is the definition of the COP and EER according to EN 14511 [8]. The third one is based on the definition of the operating curves of the heat pump, according also to the EnergyPlus software.

The common platform then leads to the different calculation methods and models. Before describing in detail the two software, it has to be underline that GeoHP-DSS is hosted in a server and hence has to be run in clouding [9], while GeoHP-Design has to be downloaded [9] and has to be installed locally in the user's PC.

\section{The Decision Support System (DSS)}

The DSS has the aim to provide a first feasibility study for the GSHP technology. This means that it suggests which technology might fit better depending on the choices of the user. As already mentioned, this tool is mainly addressed to non-technical users for facilitating their choice and make them more aware about geothermal energy solutions.

The main scheme for the DSS is shown in Figure 2. The inputs of the user are quite simple and few (Figure 3). Based on the input the calculation procedure allows to estimate the energy demand for heating/cooling and Domestic Hot Water (DHW). Different types of heat pumps are considered in the calculations and some of them are not considered due to the operating conditions (e.g. too high temperature of the terminal like in the case of radiators). Based on the COP/EER of the heat pumps, on the energy needs of the buildings, the type of ground and undisturbed temperature (both defined by the location) four types of GHEs are considered: two usual types (i.e. single $U$ and double $U$ ) and the two proposed solutions investigated in the project, i.e. the helical and the co-axial heat exchanger. The user has to define also if there is any available RES solution for the building (solar thermal, photovoltaics or wind turbine). Based on the available space around the building, the ASHARE method [2] allows to calculate the overall length of the 
GHEs in the different combinations. Based on the energy performance of the system, on the installation costs and on the running costs the results are calculated for each solution.

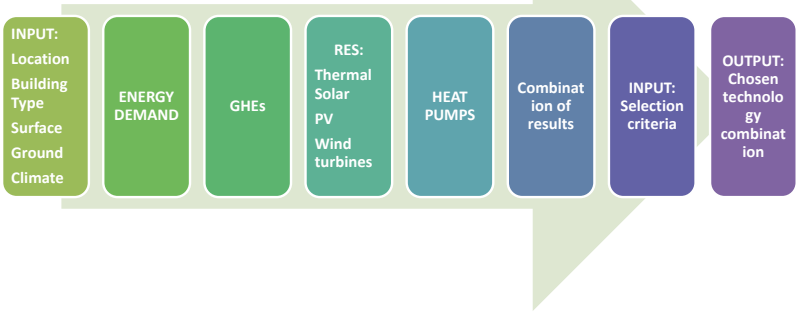

Fig. 2. Scheme of the main simplified architecture of the DSS

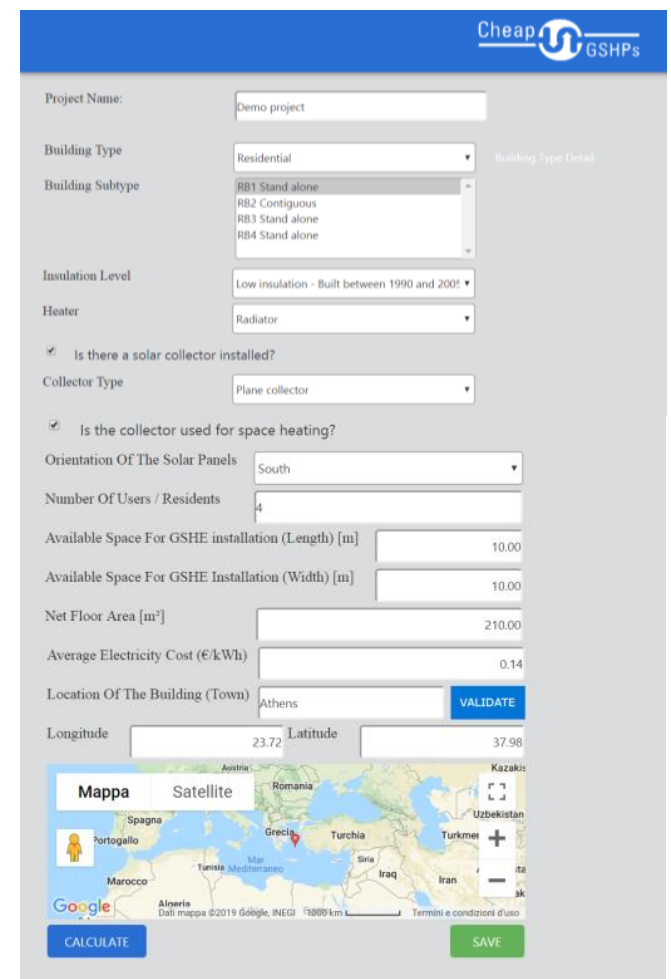

Fig. 3. Input screenshot of the DSS

At this point the user has to define his/her main interest, i.e. if he/she is interested more in environmental aspects or in economic issues. This hierarchy process is summarised in Figure 4 where the user has to choose among the different weighing criteria. Based on the selected criteria the DSS provides a classification among the different combination of possible solutions. The results are qualitative, due to the simplifications which are underpinning the tool. These simplifications are not related to the calculation procedures but rather due to the generic input which are required in order to make the tool available and usable to all types of users. This means that the input are generic and might be affected by mistake, especially related to the ground properties and thermal characteristics definition. As a matter of fact the ground properties are usually quite difficult to obtain. The database of the ground is simplified and until no pan-European maps are available with detailed values, the tool can provide only first estimations which are anyway useful for a first screening of the problem.

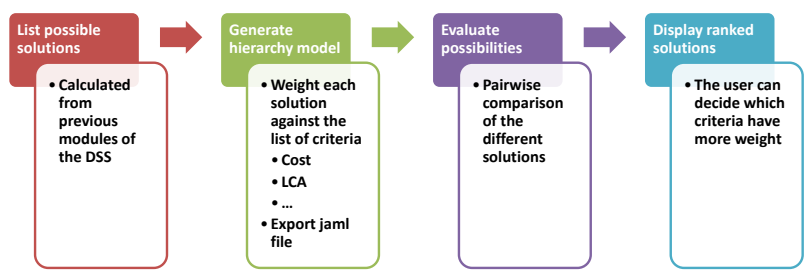

Fig. 4. Scheme of the analytic hierarchy process of the DSS

\section{The design tool}

The Design Tool has been developed based on two different calculation methods, one named simplified and one more detailed:

- The simplified model is based on the very wellknown theory of linear source method. This is known also as ASHRAE method, which has been first introduced by Kavanaugh and Rafferty [10] and then has been adopted extensively in the ASHARE Handbook [2]. Nowadays it represents the most used smart method for sizing GSHEs field.

- The detailed model (named CaRM, CapacityResistance Method) has been introduced by De Carli et al. [3] and then has been further developed by Zarrella and De Carli to make it more general, flexible and more close to the thermal behaviour of the real sys-tem by the use of dynamic calculation, as will be shown afterwards [11], [12].

\subsection{Common input}

The general frame of the Design Tool (Figure 5) allows to provide the same common input for the two methods (DSS Tool and Design Tool). The main parameters to be defined are: the climatic conditions, the energy profile of the building, the ground thermal properties and the GSHE type (geometry and thermal characteristics of the materials).

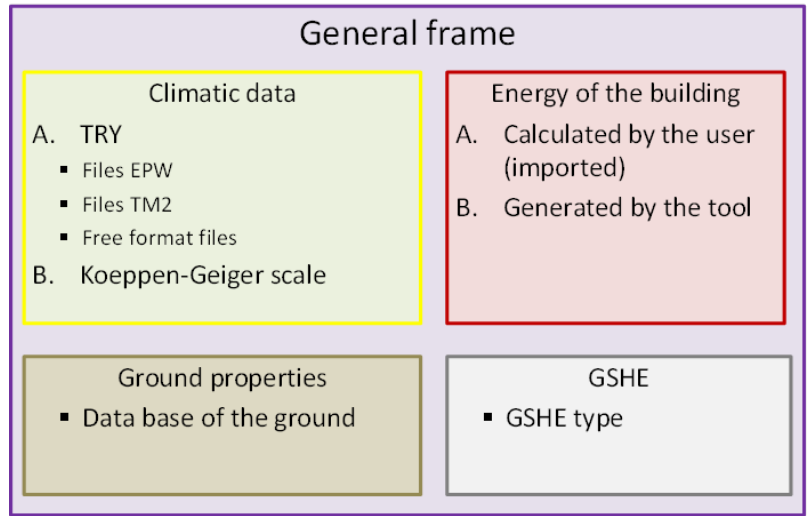

Fig. 5. General common frame for input of both the simplified method and the detailed method 


\section{Climatic data}

The starting point is the definition of the climatic conditions. A database of climatic data has been built up, including all European locations of Eenergyplus and 6 climatic files of Meteonorm. The database can be enlarged by the user by adding other EPW files or other Meteonorm TM2 files. It is also possible to import CSV files defined by the user.

\section{Building load}

Then it is needed to input the building energy demand as shown in Figure 6 where a screenshot of the program is shown. The building loads can be defined by the user or can be calculated by the tools, based on the database of buildings (the same as the one included in the DSS database). The option to make the tool to calculate the load profiles has been introduced to allow the designer to estimate with a first sizing of the plant based on very simple information (i.e. the floor area of the building and the type of envelope). This is defined in the upper part of the screen.

If the building loads are defined by the user, for the simplified model the user has to input the twelve energy needs (positive for heating, negative for cooling) and the peak power for heating and cooling, according to the ASHRAE method. For the detailed method. These data are inserted in the lower left side of the screenshot.

If the detailed method is used, the user has to upload the hurly loads of the building based on dynamic simulations. This means that an excel file with 8760 values is expected (positive values for heating, negative values for heating).

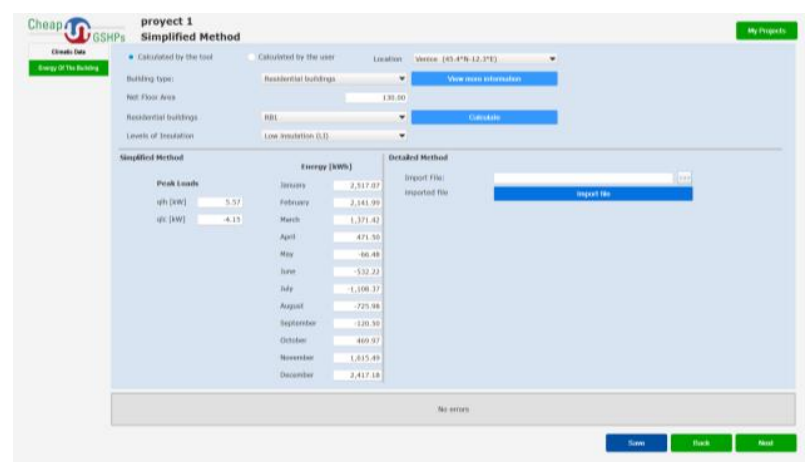

Fig. 6. Definition of the building loads

\section{Ground properties}

The user has to enter the ground properties, listing the different layers with their thickness, thermal conductivity, density and specific heat (Figure 7). A database of the thermal characteristics of the ground is present; the library can be extended by the user.

\section{Ground heat exchanger definition}

The user has then to define the heat exchanger characteristics (Figure 8). The required input are the heat exchanger geometry, i.e. the diameter of the probe, the pipes dimensions and position, the pipe thermal conductivity, the grouting thermal characteristics (density, specific heat and thermal conductivity) if any. Four types of ground heat exchangers can be defined: single $U$, double $U$, helical and co-axial. For each of them a suitable subroutine allows to calculate the thermal resistance of the borehole, which can be further changed by the user (e.g. due to the results of a TRT).

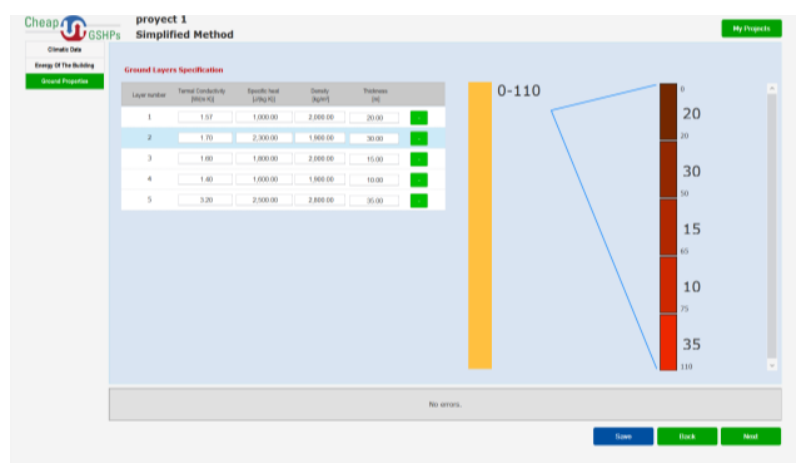

Fig. 7. Definition of the ground properties

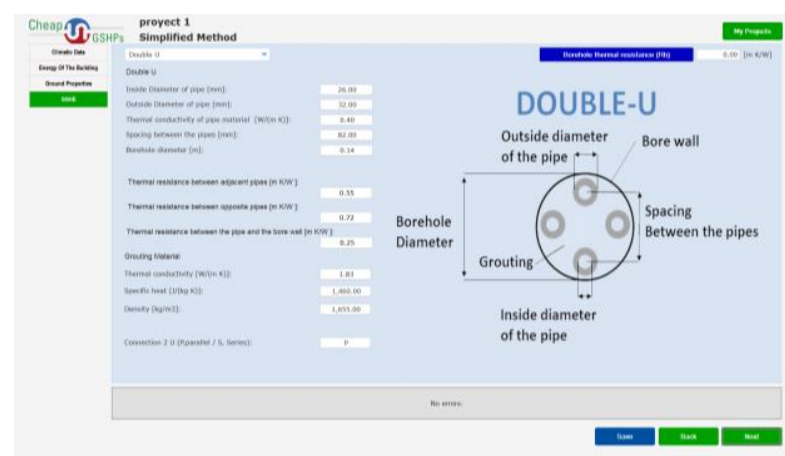

Fig. 8. Definition of the ground heat exchanger characteristics

Once inserted the GHE type, the user can decide if to proceed with a simplified calculation or with a detailed dynamic simulation.

\subsection{Simplified method (ASHRAE) \\ Heat pump definition}

The first step is to define the characteristics of the heat pump. The needed values to insert are the usual defined values of the heat pump, i.e. the return temperature from the GHEs and the supply temperature to the building. The temperature difference in the building loop and the ground loop set to $5^{\circ} \mathrm{C}$ and $3^{\circ} \mathrm{C}$ as default can be changed by the user.

For the heating conditions, the needed values to insert are the design conditions, i.e. the minimum water temperature in the ground loop and the supply temperature for the building (Figure 9). By clicking on the bottom right button "COP Declared Values" the values according to EN 14511 [8] are defined in a library which can be expanded by the user. Once set the COP declared by the manufacturer, a routine allows to calculate the COP in design conditions accordingly. The seasonal COP is also calculated based on the average temperature of the ground, calculated, as default, as average between the design temperature and the 
undisturbed temperature of the ground. This value can also be changed.

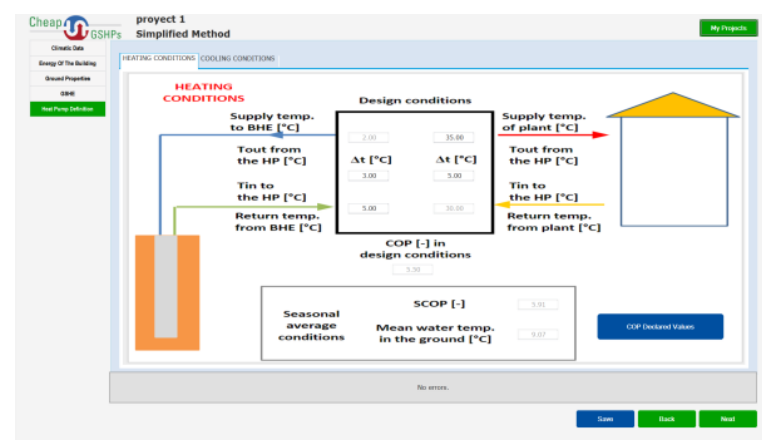

Fig. 9. Definition of the COP characteristics of the heat pump

For the cooling conditions, the needed values to insert are the design conditions, i.e. the maximum water temperature in the ground loop and the supply temperature for the building (Figure 10). By clicking on the bottom right button "EER Declared Values" the values defined according to EN 14511 [8] are defined in a library which can be expanded by the user. Once set the EER declared by the manufacturer, a routine allows to calculate the EER in design conditions accordingly. The seasonal EER is also calculated based on the average temperature of the ground, calculated, as default, as average between the design temperature and the undisturbed temperature of the ground. This value can also be changed.

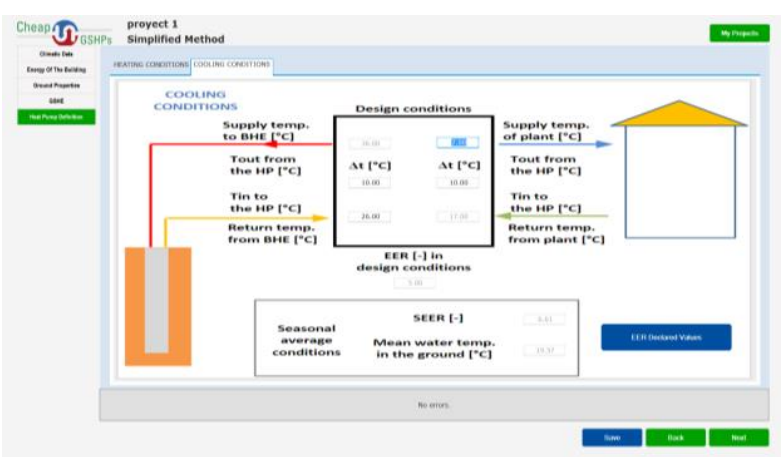

Fig. 10. Definition of the EER characteristics of the heat pump

\section{GHE field sizing}

The next step is to estimate the overall GHEs field. In this screen (Figure 11) by clicking on the button "Calculate", the first two values $\mathrm{L}_{h}$ and $\mathrm{L}_{c}$ are shown. These two values represent the ideal overall length of the GHEs field in ideal conditions (linear source model without interference between boreholes). Afterwards looking at the overall length in heating conditions $\left(\mathrm{L}_{h}\right)$ and the overall length in cooling conditions $\left(\mathrm{L}_{\mathrm{c}}\right)$ the designer will choose among the maximum (GHEs field which can cover both heating and cooling needs), the minimum (hybrid solution with dual source) or an intermediate value. Based on the choice of the overall length, the designer has also to input the GHEs field in detail by choosing the number of probes and their distribution in the ground. Based on the pattern of the field of GHEs chosen, the designer has to insert the overall depth of the drilling and the types of probes (if they are adjacent to one, two, three or four boreholes). Taking into account the types of GHEs, by clicking in the button "Optimization" the final calculation will be carried out having as a result the overall length for the GHEs field and the penalty factor.

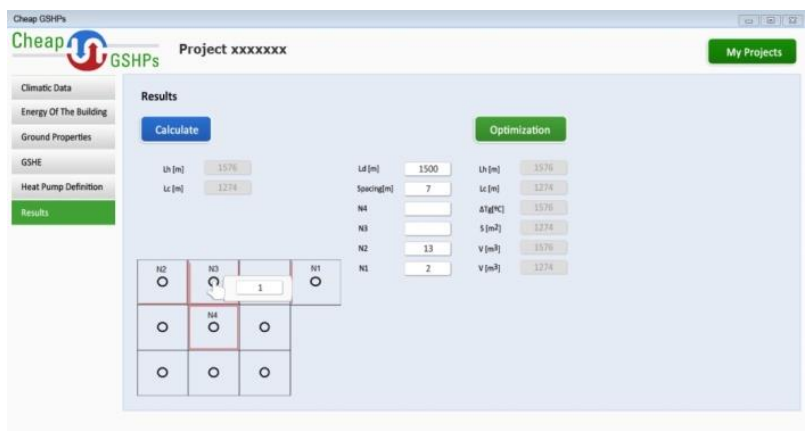

Fig. 11. Sizing of the GHEs field in the simplified method

\subsection{Detailed method (CaRM)}

\section{Backgrounds of the model}

In the detailed calculation method the model CaRM takes into account the heat exchange between the atmosphere and the ground in the so called "Surface zone", the borehole and the surrounding ground in the so called "Borehole zone" and the heat transfer below the heat exchanger in the so called "Deep zone" (Figure 12).

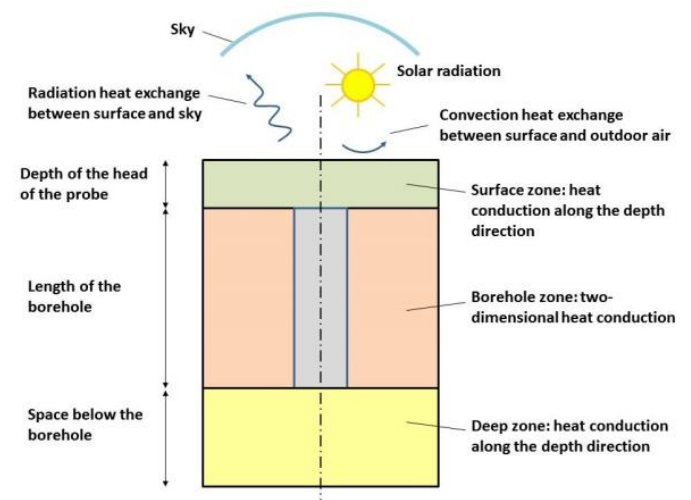

Fig. 12. General subdivision of the ground for the calculations

In the main zone where the GHE is located the heat exchange is evaluated through a network of resistances and capacities both in radial and axial direction (Figure 13.a and 13.b). 


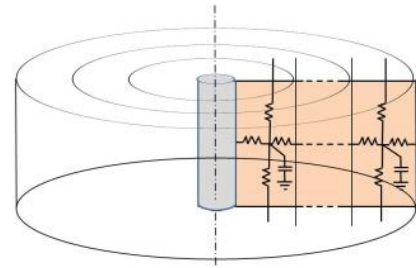

a

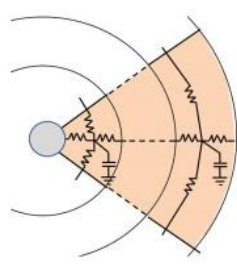

b
Fig. 13. Model for the heat exchange between the borehole and the ground in the borehole zone

In the "Surface zone" (Figure 14.a) and in the "Deep zone" (Figure 14.b) the heat exchange is one dimensional in the axial direction. In this way it is possible to simulate any kind of probe, considering also more superficial GHEs.

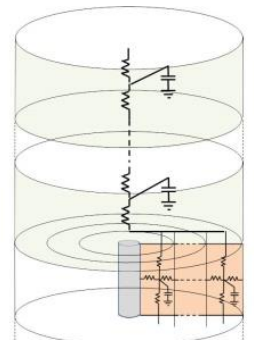

A

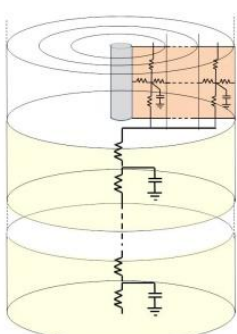

b
Fig. 14. Model for the heat exchange above (a) and below (b) the GHEs

As for the GHE geometry and thermal characteristics the thermal model is based on the assumption of two regions: the core (which is the central zone between the pipes) and shell (which is the external part between the pipes and the borehole wall). This means that all types of GHEs (single U, double U, helical, co-axial) can be divided into two zones, one with an internal resistance and a capacity (the core) and one with the external resistance shell. As an example for explaining the approach a double $U$ probe and a co-axial probe are shown in Figures 15 and 16 respectively. The model takes into account also the circulation of the water, i.e. a detailed balance of the heat transfer and mass transfer is considered for each slice of the borehole, including the thermal inertia of the water inside the pipes.

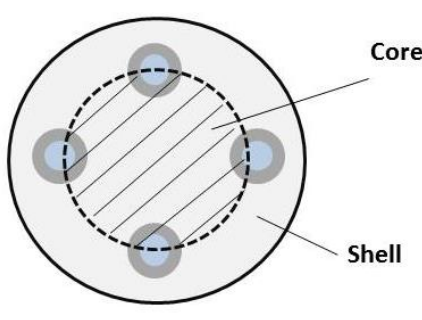

$\mathbf{a}$

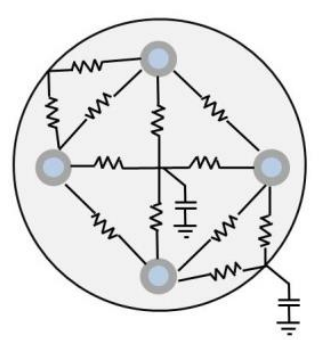

b
Fig. 15. Model of the heat transfer within the GHE: example for the double U probe.

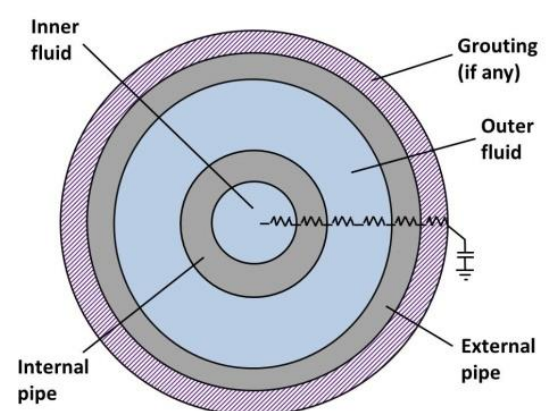

Fig. 16. Model of the heat transfer within the GHE: example for the double co-axial probe.

Definition of the input in the first screenshot (properties of surface, climate and fluid in the ground loop)

In the first screenshot (Figure 17) in the bottom part on the left there are the input related to the simulation time (time step, duration of the simulation).

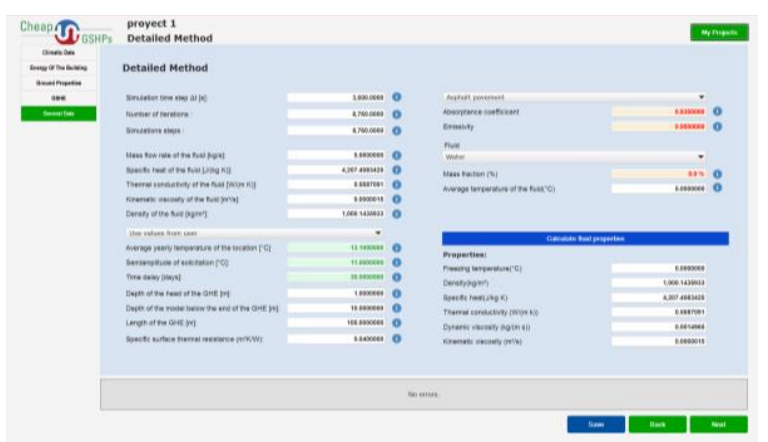

Fig. 17. First screenshot of the detailed calculation method CaRM

In the central part on the left there is the required definition of the thermal characteristics of the fluid in the ground loop, i.e. the mass flow rate, the thermal conductivity, the density, specific heat and viscosity. In the bottom right part of the screen it is possible to calculate the thermal characteristics of the fluid.

In the bottom left part of the screen the temperature of the outdoor environment and the corresponding sinusoidal solicitation. Moreover it is specified the dimension of the "Superficial zone", the "Borehole zone" and the "Deep zone".

In the right upper part of the screen the characteristics of the ground (absorption coefficient and emissivity) for the heat exchange due to solar radiation and infrared radiation of the ground surface. The possibility to choose between pure water or anti-freezing mixtures are also considered.

Definition of the input in the second screenshot (mesh) In the second screen (Figure 18) the subdivision of the ground into regions is described. The number of annular regions means how many zones along the radius are defined. The expansion coefficient allows to have the same spacing among annular regions (expansion coefficient equal to 1), a variable spacing with smaller annular regions close to the axis of the borehole (expansion coefficient $>1$ ), a variable spacing with larger regions close to the axis of the borehole 
(expansion coefficient $<1$ ). Based on previous calculations and sensitivity analyses the recommended coefficient can be equal to 1.2. According to Figure 4 the ground is subdivided vertically into three zones: the surface zone (above the GSHE), the zone below the GSHE and the zone below the GSHE. The number of sublayers is the subdivision into elements of the regions in vertical direction.

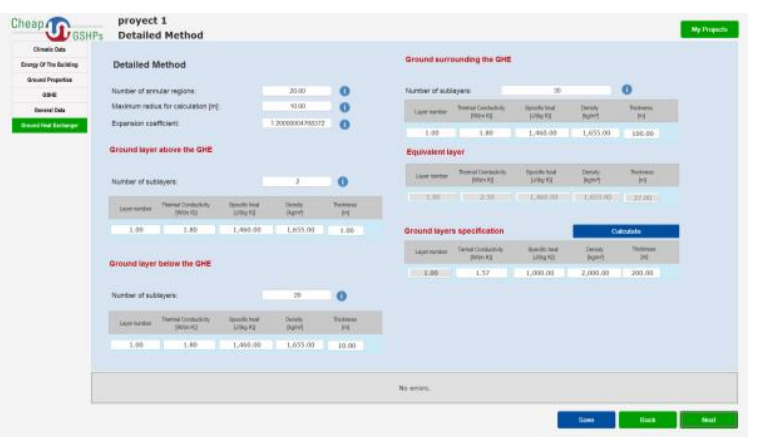

Fig. 18. Second screenshot of the detailed calculation method CaRM

Definition of the input in the third screenshot (geometry of the GHEs field)

In the third screen (Figure 19) of the detailed calculation method the GSHE field has to be defined by the user. The detailed method differs to the simplified one. In the simplified one (ASHRAE), once defined the maximum (in summer) and minimum (in winter) temperatures, the overall length is calculated. In the numerical method CaRM the length has to be fixed and the calculation provides the temperatures over time. If the temperatures do not fit in the defined thresholds, the user has to change the length and/or the geometry of the GSHE field.

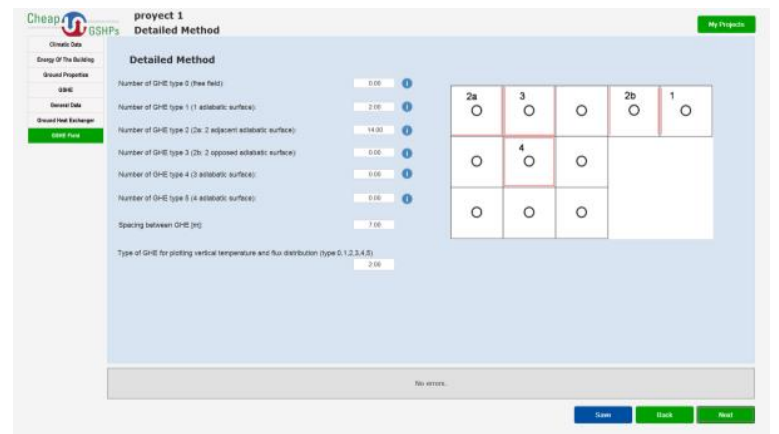

Fig. 19. Third screenshot of the detailed calculation method CaRM

Definition of the input in the fourth screenshot (heat pump definition)

In the last screen the heat pump is defined in a more detailed way with respect to the simplified method. Specific performance curves of the heat pump have to be defined according to the Energy Plus model (Figure 20).

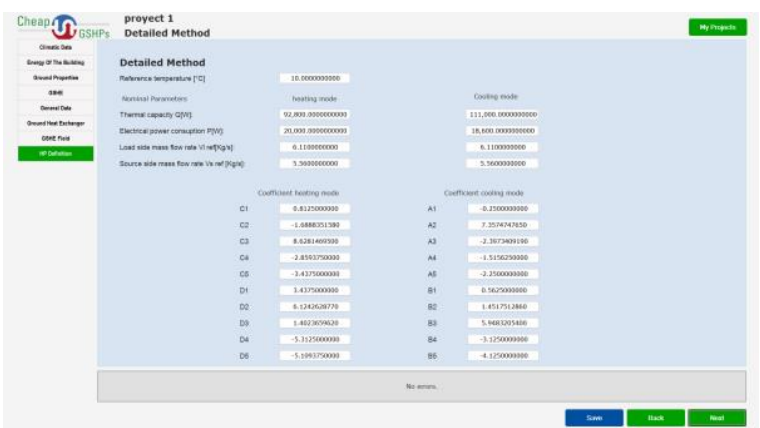

Fig. 20. Fourth screenshot of the detailed calculation method CaRM

\section{Results of the detailed calculation model}

The model solves the balance in each node. The results of the detailed calculation model may be shown as supply and return temperatures of the fluid in the ground loop for each time step. Also the temperature at a certain distance of the probe may be given as result. In Figure 21 an example of possible output is shown.

Since the detailed calculation needs several attempts to find the wanted solution, it is usually worthwhile to first start a preliminary simulation with the ASHRAE method in order to have an idea on the number of boreholes and possible geometry. Once made the first analysis with the ASHRAE method, the CaRM model can be set up and run.

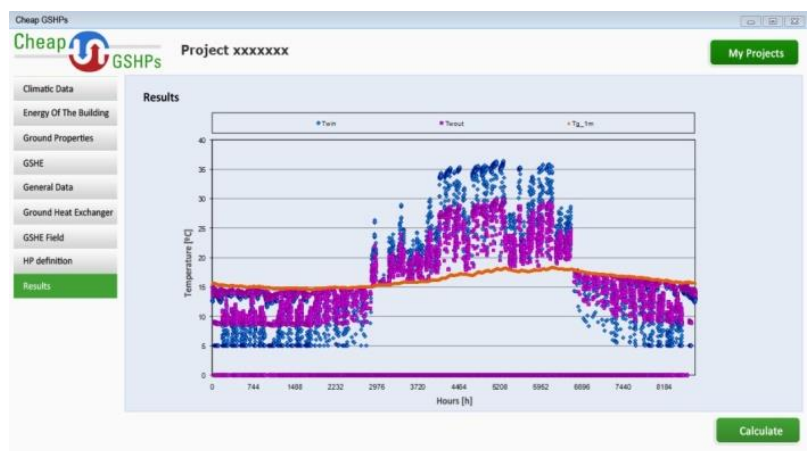

Fig. 21. Screenshot of results of the detailed calculation method CaRM

\section{Discussion and conclusions}

The paper presents the two software which have been produced in the H2020 Cheap-GSHPs project. The aim of the two tools is to destroy barriers and make more feasible the GSHPs, facilitating decisions to stakeholders. In this way people will be more sensible to GSHPs, since they will be aware about the environmental benefits of GSHPs compared to other solutions. These particular goals are related to the DSS software which is a simplified tool where each user even without any particular background will be able to get more confident with the GSHP technology.

The DSS is based on different databases which have been built up during the project. The program generates 
different solutions and makes a classification based on hierarchies defined by the user.

For facilitating the design to technicians and to get designers closer to GSHPs, a Design tool has been developed. The tool allows to make two possible calculations. The first possibility is to use the analytical solution based on the ASHRAE method. The tool allows to have a user-friendly interface which allows to make the calculation in a simple way.

The second possibility is to use the mathematical model CaRM, a detailed lumped model which allows to make dynamic simulations of the ground. This tool allows to estimate any type of GHE: single $\mathrm{U}$, double $\mathrm{U}$, helical and co-axial. The detailed calculation method allows also to take properly into account the thermal inertia of the grouting as well as the heat capacity of the water flowing inside the GHEs.

The tools are freely available and they will allow to destroy the barriers in the diffusion of the GSHPs market.

\section{Acknoledgements}

This work has received funding from the European Union's Horizon 2020 research and innovation program under grant agreement No. 657982.

\section{References}

[1] E. Turban; R. K. Rainer; R. E. Potter, Introduction to Information Technology, 3rd Edition, (2004) ISBN 978-0-47134780-4.

[2] S.P. Kavanaugh, K. Rafferty, Ground-source Heat Pumps Design of Geothermal System for Commercial and Institutional Buildings ASHRAE Applications Handbook, Atlanta, GA, US (1997)

[3] M. De Carli, M. Tonon, A. Zarrella, R. Zecchin. A computational capacity resistance model $(\mathrm{CaRM})$ for vertical ground-coupled heat exchangers, Ren. En 35, pages 1537-1550 (2010).

[4] J. Müller, A. Galgaro, G. Dalla Santa, M. Cultrera, C. Karytsas, D. Mendrinos, S. Pera, R. Perego, N. O’Neill, R. Pasquali, J. Vercruysse, L. Rossi, A. Bernardi, D. Bertermann, Generalized Pan-European Geological Database for Shallow Geothermal Installations, Geosciences 8(1) 32 (2010). doi:10.3390/geosciences8010032.

[5] G. Dalla Santa, F. Peron, A. Galgaro, M. Cultrera, D. Bertermann, J. Mueller, A. Bernardi, Laboratory Measurements of Gravel Thermal Conductivity: An Update Methodological Approach, En. Proc. 125, pag. 671-677, (2017). doi: 10.1016/ j.egypro.2017.08.287.

[6] M. De Carli, A. Bernardi, M. Cultrera, G. Dalla Santa, A. Di Bella, G. Emmi, A. Galgaro, S. Graci, D. Mendrinos, G. Mezzasalma, R. Pasquali, S. Pera, R. Perego, A. Zarrella., A Database for Climatic Conditions around Europe for Promoting GSHP Solutions, Geosciences (Switzerland) 8(2), (2018)

[7] B. Badenes, M. Bellieardi, A. Bernardi, M. De Carli, M. Di Tuccio, G. Emmi, A. Galgaro, S. Graci, L. Pockelè, A. Vivarelli, S. Pera, J. F. Urchueguía, A. Zarrella, Definition of Standardized Energy Profiles for Heating and Cooling of Buildings, In Proceedings of the 12th REHVA World Congress;
Heiselberg, Per Kvols: Aalborg, Denmark, Vol. 6, (2016).

[8] EN 14511. Air conditioners, liquid chilling packages and heat pumps for space heating and cooling and process chillers, with electrically driven compressors.

[9] http://cheap-gshp.eu/ (last seen 15/01/2019)

[10] S.P. Kavanaugh, K. Rafferty, Ground-source Heat Pumps Design of Geothermal System for Commercial and Institutional Buildings ASHRAE Applications Handbook, Atlanta, GA, US, (1997).

[11] A. Zarrella, M. Scarpa, M. De Carli. Short time step analysis of vertical ground-coupled heat exchangers: the approach of CaRM, Ren. En., Vol. 36, n. 9, pages 2357-2367, (2011).

[12] A. Zarrella, M. De Carli. Heat transfer analysis of short helical borehole heat exchangers, App. En., Vol. 102, n. 2, pages 14771491, (2013). 